\title{
Thermodynamic properties of graphene bilayers
}

\author{
Carlos P. Herrero $\odot^{*}$ and Rafael Ramírez \\ Instituto de Ciencia de Materiales de Madrid, Consejo Superior de Investigaciones Científicas (CSIC), \\ Campus de Cantoblanco, 28049 Madrid, Spain
}

(Received 26 September 2019; revised manuscript received 16 December 2019; published 8 January 2020)

\begin{abstract}
Thermodynamic properties of graphene bilayers are studied by path-integral molecular dynamics (PIMD) simulations, considering quantization of vibrational modes and anharmonic effects. Bilayer graphene has been studied at temperatures between 12 and $1500 \mathrm{~K}$ for zero external stress, using the LCBOPII effective potential. We concentrate on the thermal expansion, in-plane and out-of-plane compressibility, and specific heat. Additional insight into the meaning of our results for bilayer graphene is obtained from a comparison with data obtained from PIMD simulations for monolayer graphene and graphite. They are also analyzed in view of experimental data for graphite. Zero-point and thermal effects on the in-plane and "real" area of bilayer graphene are studied. The thermal expansion coefficient $\alpha_{x y}$ of the in-plane area is negative at low temperatures and positive for $T \gtrsim 800 \mathrm{~K}$. The minimum $\alpha_{x y}$ is $-6.6 \times 10^{-6} \mathrm{~K}^{-1}$ at $T \approx 220 \mathrm{~K}$. Both in-plane $\left(\chi_{x y}\right)$ and out-of-plane $\left(\chi_{z}\right)$ compressibilities of graphene bilayers are found to increase for rising temperature, and turn out to be lower than that corresponding to monolayer graphene and higher than those found for graphite. At $300 \mathrm{~K}$, we find for the bilayer $\chi_{x y}=9.5 \times 10^{-2} \AA^{2} / \mathrm{eV}$ and $\chi_{z}=2.97 \times 10^{-2} \mathrm{GPa}^{-1}$. Results for the specific heat obtained from the simulations are compared with those given by a harmonic approximation for the vibrational modes. This approach is noticeably accurate at temperatures lower than $200 \mathrm{~K}$.
\end{abstract}

DOI: 10.1103/PhysRevB.101.035405

\section{INTRODUCTION}

Graphene bilayers have attracted great interest in last years after the finding that they present unconventional superconductivity when stacking both sheets twisted relative to each other by a small angle [1-3]. It has been also recently noticed the existence of Mott-like insulator states in these materials, for the appearance of localized electrons in the superlattice corresponding to a moire pattern [4,5]. Moreover, twisted graphene bilayers display magnetic properties which can be externally controlled by an applied bias voltage $[6,7]$. Graphene bilayers are known to show ripples and out-ofplane deformations similar to the monolayers [8], causing a departure from planarity which is believed to be a relevant mechanism for electron scattering [9].

From a basic point of view, understanding the thermodynamic properties of two-dimensional (2D) systems in threedimensional (3D) space has been along the years a continuous objective in the field of statistical physics $[10,11]$. This problem has been mainly treated in connection with soft condensed matter and biological membranes [12,13], whose complexity makes it very demanding to devise microscopic models built on realistic interatomic interactions. Graphene bilayers are a well-controlled instance of crystalline membranes formed by two atomic sheets, for which an atomic-level description is possible, allowing for a deep insight into the physical properties of this kind of systems [14-18]. Moreover, graphene shows us as a suitable material to study the thermodynamic stability of 2D crystals, which has been long discussed and

*ch@icmm.csic.es can be related to anharmonic coupling between in-plane and out-of-plane vibrational modes [17,19].

Various kinds of atomistic simulations have been employed to study finite-temperature properties of graphene [20-24]. In most of them, $\mathrm{C}$ atoms were considered as classical particles, but the Debye temperature of graphene for out-of-plane vibrational modes is $\Theta_{D}^{\text {out }} \gtrsim 1000 \mathrm{~K}$ and higher for in-plane modes [25]. This indicates that the influence of quantum fluctuations on physical properties should be appreciable even for $T$ much higher than room temperature.

Some works have presented path-integral-type simulations, which allow one to study thermal and quantum fluctuations at finite temperatures. This type of simulations have been carried out for graphene monolayers to study structural and thermodynamic properties of this material [26-29]. In addition to this, nuclear quantum effects have been analyzed earlier by means of a combination of density-functional theory and a quasiharmonic approximation for vibrational modes in this crystalline membrane [30,31].

The thermal behavior of monolayer graphene has been studied by means of path-integral simulations [28], with particular emphasis on low temperatures. In this paper, we extend that analysis to graphene bilayers, where new aspects are expected to appear due to interlayer interactions, and the associated coupling between atomic displacements of both layers in the out-of-plane direction.

We employ the path-integral molecular dynamics (PIMD) method to study thermodynamic properties of graphene bilayers at temperatures between 12 and $1500 \mathrm{~K}$. Simulation cells of different sizes are considered, as finite-size effects have been found earlier to be important for some equilibrium properties of graphene [24,27,32]. We analyze the thermal 
behavior of the sheet surface in graphene bilayers, considering the difference between real and in-plane area. We put special attention on the temperature dependence of the thermal expansion, compressibility, and specific heat $c_{p}$. In particular, low-temperature results of the simulations for $c_{p}$ are compared with the prediction of a harmonic approximation for the vibrational modes.

The paper is organized as follows. In Sec. II, we describe the computational method used in the simulations. In Sec. III, we present results for the real and in-plane areas, as well for the so-called excess area of graphene bilayers. The thermal expansion is discussed in Sec. IV, and the compressibility (in-plane and out-of-plane) is analyzed in Sec. V. In Sec. VI, we present results for the specific heat, and in Sec. VII, we summarize the main results.

\section{COMPUTATIONAL METHOD}

\section{A. Path-integral molecular dynamics}

Here we employ PIMD simulations to study structural and thermodynamic properties of graphene bilayers as a function of temperature. This method, based on the Feynman pathintegral formulation of statistical mechanics [33], is now a well-established nonperturbative approach to investigate finite-temperature properties of many-body quantum systems. In the applications of this computational technique to numerical simulations, each quantum particle (here atomic nucleus) is represented as a group of $N_{\mathrm{Tr}}$ beads (the so-called Trotter number), behaving like classical particles disposed to form a ring polymer [34-36].

In actual simulations of condensed matter using the pathintegral method, the configuration space of the classical isomorph is explored by means of molecular dynamics or Monte Carlo sampling. In this paper, we use molecular dynamics, as we have found that our computing codes are more effectively parallelizable with this procedure. We note that the dynamics in this kind of PIMD simulations is artificial, in the sense that it does not reproduce the dynamics of the actual quantum particles under consideration. Nevertheless, it is very efficient to sample the many-body configuration space, giving precise results for time-independent equilibrium properties of the quantum system.

We describe the interatomic interactions in graphene with a long-range carbon bond-order potential, the so-called LCBOPII [37], which has been employed earlier to carry out classical simulations of carbon-based systems, such as diamond [37], graphite [37], and liquid carbon [38]. It has been more recently applied to study graphene [20,24,39], with particular emphasis on its mechanical properties [40,41]. The LCBOPII potential has been also used to perform PIMD simulations of graphene monolayers [27] and bilayers [42], which has allowed an assessment of quantum effects by comparing with results of classical simulations. In this paper, according to earlier simulations $[27,41,43]$, the original LCBOPII parametrization has been slightly changed to increase the zero-temperature bending constant $\kappa$ of a graphene monolayer from $0.82 \mathrm{eV}$ to a more realistic value of $1.49 \mathrm{eV}$, closer to experimental data and $a b$ initio calculations [44]. The interlayer interaction is the same as that employed in earlier simulations of bilayer graphene with this effective potential $[39,42]$. Thus the interlayer binding energy for the minimumenergy configuration with $\mathrm{AB}$ stacking is $25 \mathrm{meV} /$ atom for bilayer graphene and $50 \mathrm{meV} /$ atom for graphite.

Our simulations of graphene bilayers have been performed in the isothermal-isobaric ensemble, where we fix the number of carbon atoms $(2 N)$, the in-plane stress (here $\left.P_{x y}=0\right)$, and the temperature $(T)$. We employed effective algorithms for carrying out PIMD simulations, as those presented in the literature $[45,46]$. Specifically, staging variables [47] were used to define the bead coordinates, and a constant temperature was attained by coupling chains of four Nosé-Hoover thermostats $[48,49]$. Another chain of four barostats was coupled to the in-plane area of the simulation box ( $x y$ plane) to yield a constant pressure $P_{x y}=0[36,45]$. The equations of motion were integrated by using the reversible reference system propagator algorithm (RESPA), which permits to consider different time steps for the integration of fast and slow degrees of freedom [50]. The time step $\Delta t$ associated to the interatomic forces was taken as $0.5 \mathrm{fs}$, which was adequate for the atomic mass and temperatures considered here. The kinetic energy was calculated by employing the virial estimator, which shows a statistical uncertainty smaller than the primitive estimator, in particular at high temperatures [45,51]. More technical details on this type of PIMD simulations are given elsewhere [45,52,53].

We have considered graphene bilayers with $\mathrm{AB}$ stacking in rectangular simulation cells including $2 N$ carbon atoms, $N$ going from 24 to 8400 . These cells had similar side lengths in the $x$ and $y$ directions $\left(L_{x} \approx L_{y}\right)$, for which periodic boundary conditions were assumed. Carbon atoms can move without restriction in the out-of-plane direction, i.e., we have free boundary conditions in the $z$ coordinate, reproducing a freestanding graphene bilayer. We considered temperatures $T$ in the range from 12.5 to $1500 \mathrm{~K}$. Given a temperature, a typical simulation run consisted of $2 \times 10^{5}$ PIMD equilibration steps and $8 \times 10^{6}$ steps for the calculation of average variables. The number of beads, $N_{\mathrm{Tr}}$, was taken proportional to $1 / T$, so that $N_{\mathrm{Tr}} T=6000 \mathrm{~K}$, which keeps a nearly constant accuracy for the results at different temperatures. To assess the magnitude of nuclear quantum effects, some classical molecular dynamics simulations of graphene bilayers have been also carried out. This corresponds in our context to setting $N_{\mathrm{Tr}}=1$. In Fig. 1, we present a top view of a configuration of bilayer graphene obtained in our simulations at $T=1000 \mathrm{~K}$. In this picture, red and black circles represent $\mathrm{C}$ atoms in the upper and lower sheets in AB stacking pattern.

For comparison with the results for graphene bilayers, we have also performed some PIMD simulations of graphite with the interatomic potential LCBOPII. For this 3D material, we used simulation cells containing $4 N$ carbon atoms (four graphene sheets), and periodic boundary conditions were assumed in the three space directions. We used cells with $N=240$ and 960 .

\section{B. Harmonic approximation}

To compare with the results of PIMD simulations for the specific heat of bilayer graphene, we will present a harmonic approximation (HA) for the lattice vibrations. This 


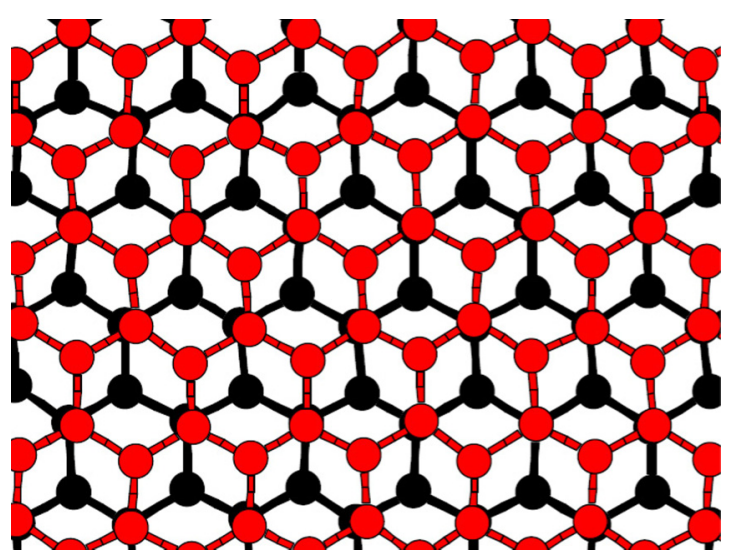

FIG. 1. Top view of an instantaneous configuration of bilayer graphene at $T=1000 \mathrm{~K}$. Red and black circles represent carbon atoms in the upper and lower sheets, respectively.

approximation turns out to be rather precise at low temperature, but anharmonicity appears for rising temperature, so that the results of the HA will increasingly deviate from those derived from the simulations. A basic assumption of the HA is that vibrational frequencies in the material do not change with temperature. Then, we take in this model the frequencies corresponding to the minimum-energy configuration of bilayer graphene, obtained from diagonalization of the dynamical matrix for the LCBOPII potential.

In a quantum HA, the vibrational energy per atom of bilayer graphene is given by

$$
E_{\mathrm{vib}}=\frac{1}{2 N} \sum_{j, \mathbf{k}} \frac{1}{2} \hbar \omega_{j}(\mathbf{k}) \operatorname{coth}\left(\frac{1}{2} \beta \hbar \omega_{j}(\mathbf{k})\right),
$$

where $\beta=1 /\left(k_{B} T\right), k_{B}$ is Boltzmann's constant, and the index $j(j=1, \ldots, 12)$ refers to the phonon bands: four branches with atomic displacements along the $z$ direction (ZA, ZO', and a twofold degenerate $\mathrm{ZO}$ band), and eight branches with in-plane displacements (LA, TA, LO, and TO, all of them twofold degenerate) [54-57]. The sum in $\mathbf{k}$ is extended to wave vectors $\mathbf{k}=\left(k_{x}, k_{y}\right)$ in the $2 \mathrm{D}$ hexagonal Brillouin zone, with $\mathbf{k}$ points spaced by $\Delta k_{x}=2 \pi / L_{x}$ and $\Delta k_{y}=2 \pi / L_{y}$ [43]. In the following, $k$ will denote the wave number, i.e., $k=|\mathbf{k}|$.

The specific heat per atom, $c_{v}(T)=d E_{\mathrm{vib}} / d T$, is given in the HA by

$$
c_{v}(T)=\frac{k_{B}}{2 N} \sum_{j, \mathbf{k}} \frac{\left[\frac{1}{2} \beta \hbar \omega_{j}(\mathbf{k})\right]^{2}}{\sinh ^{2}\left[\frac{1}{2} \beta \hbar \omega_{j}(\mathbf{k})\right]} .
$$

Increasing the system size $N$ causes the appearance of vibrational modes with longer wavelength $\lambda$. In fact, one has for the phonons an effective cutoff $\lambda_{\max } \approx L$, with $L=\left(L_{x} L_{y}\right)^{1 / 2}$, and the minimum wave number is $k_{0}=2 \pi / \lambda_{\max }$, which means that $k_{0}$ scales as $N^{-1 / 2}$.

At low temperature, one can obtain an analytic dependence of the specific heat by assuming a continuous model for frequencies and wave numbers, which allows to replace sums by integrals in Eqs. (1) and (2). This is explained in Sec. VI and Appendix B.

\section{EXCESS AREA}

In our PIMD simulations in the isothermal-isobaric ensemble, we fix the applied stress in the $(x, y)$ plane (here $\left.P_{x y}=0\right)$, as indicated in Sec. II A, thus allowing for changes in the in-plane area of the simulation cell. Carbon atoms can freely move in the $z$ coordinate (out-of-plane direction), which means that at $T>0$ the real surface of a graphene layer will not be planar, with an area (in 3D space) larger than that of the simulation cell in the $(x, y)$ plane. The difference between the real area $A$ and in-plane area $A_{p}$ has been discussed in the literature for biological membranes [12,58,59] and more recently for crystalline membranes such as graphene [41]. It has been shown that values of the compressibility may be very different when they are related to $A$ or to $A_{p}$ [41].

A precise distinction between both areas is important to explain some thermodynamic properties of $2 \mathrm{D}$ materials. Thus the area $A_{p}$ is the conjugate variable to the in-plane stress $P_{x y}$ in the isothermal-isobaric ensemble used here, while the area $A$ is conjugate to the usually called surface tension [10]. In recent years, Nicholl et al. [60,61] have found that some experimental techniques are sensitive to properties related to the real area $A$, and other procedures can be adequate to study variables associated to the in-plane area $A_{p}$.

In our PIMD simulations, we have calculated the real area $A$ of the graphene layers by a triangulation based on the atomic positions [41,42]. In the following, $A$ and $A_{p}=L_{x} L_{y} / N$ will refer to the real and in-plane area per atom, respectively. The areas $A$ and $A_{p}$ coincide for strictly planar graphene layers, a condition met in the classical zero-temperature limit, while for $T>0$ one has $A>A_{p}$. Even for $T \rightarrow 0, A$ and $A_{p}$ are not exactly equal when nuclear quantum effects are taken into account, due to zero-point motion in the out-of-plane direction $[28,42]$. For graphene monolayers and bilayers, it turns out that both areas present qualitatively different temperature dependencies: the in-plane area $A_{p}$ displays negative thermal expansion in a large temperature region, while the real area $A$ does not show that behavior $[27,40,42]$. Moreover, $A_{p}$ depends on the system size, whereas $A$ is rather insensitive to it.

In the results of our PIMD simulations of graphene bilayers, we observe that the in-plane area $A_{p}$ decreases as $T$ rises in the region from $T=0$ to temperatures of about $800 \mathrm{~K}$, where it reaches a minimum, and then it grows at higher $T$. $A_{p}(T)$ presents a minimum for all considered system sizes. This minimum becomes deeper and smoothly shifts to higher temperatures as $N$ increases, converging to a value $T_{m}=850( \pm 50) \mathrm{K}$ for the largest cells considered here. In Fig. 2, we display the dependence of $T_{m}$ on system size, where solid circles indicate results of PIMD simulations for bilayer graphene. For comparison, we have also plotted data for monolayer graphene (squares) and graphite (diamonds), also derived from PIMD simulations with the LCBOPII potential model. Dashed lines are polynomial fits to the data points. We observe that the convergence of $T_{m}$ to its large-size limit is slower for bilayer graphene than for graphite, but faster than in the case of an isolated monolayer. This is due to the larger out-of-plane vibrational amplitudes in the monolayer, which are reduced in the bilayer, and are even less for graphite.

Both areas $A$ and $A_{p}$ derived from PIMD simulations show a temperature derivative which approaches zero as $T \rightarrow 0$, 


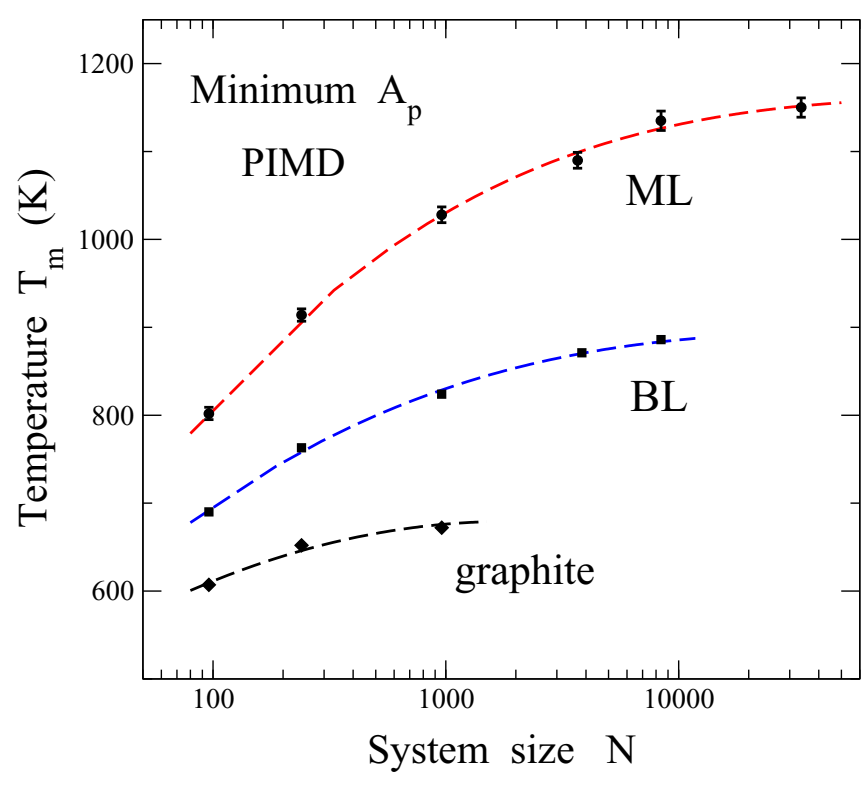

FIG. 2. Temperature $T_{m}$ corresponding to the minimum in-plane area $A_{p}$ as a function of system size. Symbols are data points derived from PIMD simulations for graphene monolayer (ML, circles), bilayer (BL, squares), and graphite (diamonds). Lines are polynomial fits to the data points. Error bars, when not displayed, are in the order or less than the symbol size.

in agreement with the third law of thermodynamics [28,62]. For $T \rightarrow 0, A$ is slightly larger than $A_{p}$, and the difference between both areas grows with temperature. Indeed $A_{p}$ is a 2D projection of the real surface on the $(x, y)$ plane, and ripples of the actual surface have larger amplitudes at higher temperatures. The difference between real and in-plane area has been called hidden area for graphene in Ref. [61], as well as excess area for fluid membranes [63,64]. In this line, for each temperature $T$, we define the dimensionless excess area, $\Omega$, of a graphene sheet as $[63,64]$

$$
\Omega=\frac{A-A_{p}}{A_{p}} .
$$

In Fig. 3, we present $\Omega$ as a function of $T$ for bilayer graphene, as derived from our PIMD simulations (solid circles). For comparison, we also display the excess area for monolayer graphene (squares) and graphite (diamonds). Dashed lines are guides to the eye. The data shown here were obtained for system size $N=960$. In the three cases, we find a lowtemperature limit $\Omega_{0}=2.0(1) \times 10^{-3}$, i.e., $A-A_{p}=5.3 \times$ $10^{-3} \AA^{2} /$ atom, due to zero-point motion in the out-of-plane direction. As a result, the excess area grows as temperature is raised, in accord with an increasing amplitude of the out-ofplane vibrational modes. This increase is lower for the bilayer than for the monolayer, and it is even smaller for graphite. Note that in a classical model $\Omega$ vanishes for $T \rightarrow 0$, as shown in Fig. 3 for the results of classical MD simulations of the bilayer (dashed-dotted line).

The excess area can be calculated in a HA for the vibrational modes, taking into account that the difference between real and in-plane area is related to the amplitude of the modes in the out-of-plane direction. For a graphene sheet, the relation

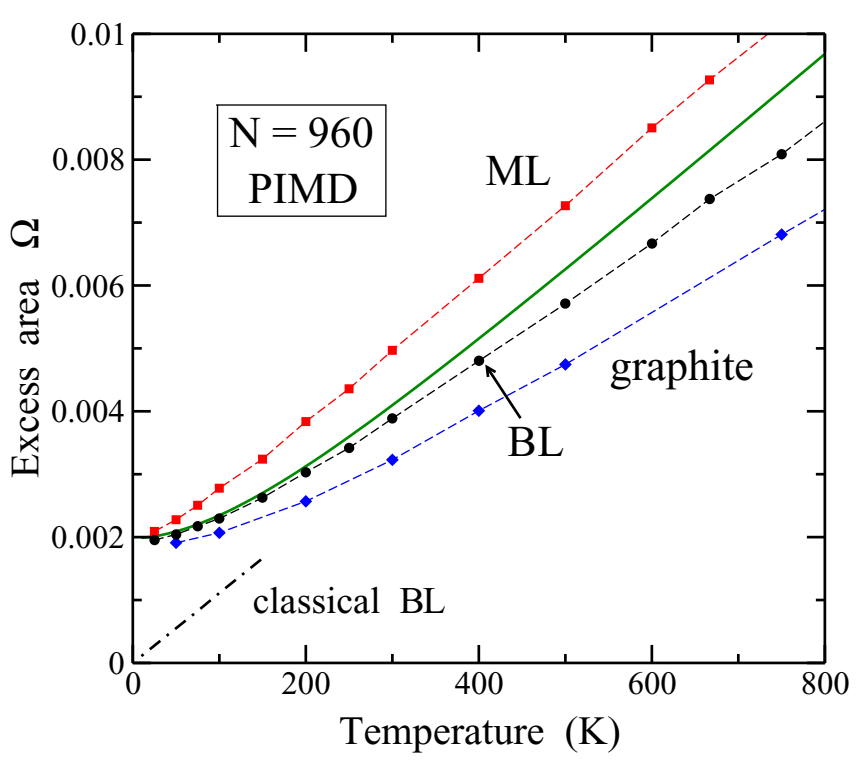

FIG. 3. Temperature dependence of the dimensionless excess area $\Omega$ as derived from PIMD simulations for system size $N=$ 960 for graphene monolayer (ML, squares), bilayer (BL, circles), and graphite (diamonds). Dashed lines are guides to the eye. Error bars are less than the symbol size. The continuous line is the HA result derived from Eq. (6) in the main text. The dashed-dotted line indicates the results of classical MD simulations for the bilayer in the low-temperature region.

between its instantaneous real area $A_{\text {inst }}$ and the in-plane area $A_{p}$ can be written in a continuous approach as $[41,58,59]$

$$
A_{\text {inst }}=\int_{A_{p}} d x d y \sqrt{1+|\nabla h(\mathbf{r})|^{2}},
$$

where $\mathbf{r} \equiv(x, y)$ is the $2 \mathrm{D}$ position and $h(\mathbf{r})$ is the distance to the mean $(x, y)$ plane of the sheet.

The difference $A_{\text {inst }}-A_{p}$ can be calculated by expanding the height $h(\mathbf{r})$ as a Fourier series with wave vectors $\mathbf{k}=$ $\left(k_{x}, k_{y}\right)$ in the 2D hexagonal Brillouin zone $[10,12,41]$ (see Appendix A). One finds

$$
A=\left\langle A_{\text {inst }}\right\rangle=A_{p}\left[1+\frac{1}{2 N} \sum_{\mathbf{k}} k^{2}\left\langle|H(\mathbf{k})|^{2}\right\rangle\right],
$$

$H(\mathbf{k})$ being the Fourier components of $h(\mathbf{r})$. Then, we have for the excess area

$$
\Omega=\frac{1}{2 N} \sum_{j, \mathbf{k}} k^{2}\left\langle\left|\xi_{j}(\mathbf{k})\right|^{2}\right\rangle,
$$

with the mean-square displacements (MSDs) in a harmonic approximation

$$
\left\langle\left|\xi_{j}(\mathbf{k})\right|^{2}\right\rangle=\frac{\hbar}{2 m \omega_{j}(\mathbf{k})} \operatorname{coth}\left(\frac{1}{2} \beta \hbar \omega_{j}(\mathbf{k})\right)
$$

For comparison with the results of our PIMD simulations, we present in Fig. 3 the excess area $\Omega$ calculated for bilayer graphene by means of Eq. (6) (solid line), considering the vibrational modes in the bands with out-of-plane displacements (ZA, ZO', and the twofold degenerate ZO). The HA yields results for the excess area close to those of the PIMD 
simulations at temperatures up to $200 \mathrm{~K}$. At higher $T$, this approximation predicts $\Omega$ values which progressively depart from those of the simulations, in accordance with an increasing departure from harmonicity of the vibrational modes.

More insight into the physical meaning of the excess area as calculated from the MSDs $\left\langle\left|\xi_{j}(\mathbf{k})\right|^{2}\right\rangle$ in Eq. (6) can be obtained by looking at the classical (high temperature) limit in Eq. (7). In this limit, the MSDs are given by $k_{B} T / m \omega_{j}(\mathbf{k})^{2}$. This means that for low-frequency acoustic modes (LA and TA) with $\omega \sim k$, the contribution to the sum in Eq. (6) is independent of $k$. However, for the flexural ZA band with a negligible effective stress $\sigma\left(\sigma \ll \kappa k^{2}\right)$, one has $\omega_{\mathrm{ZA}} \approx$ $\sqrt{\kappa} k^{2}$, so that $k^{2}\left\langle\left|\xi_{j}(\mathbf{k})\right|^{2}\right\rangle \sim k^{-2}$, which makes the contribution of the flexural band the dominant part in the sum in Eq. (6). Taking into account that the minimum wavenumber $k_{0}$ scales with cell size as $k_{0} \sim N^{-1 / 2}$ (see Sec. II B), its contribution to $\Omega$ scales linearly with $N$, eventually diverging in the thermodynamic limit. This divergence is eliminated in the presence of an effective stress (even small) $\sigma$. Note that in the classical limit $\Omega$ vanishes for $T \rightarrow 0$, at odds with the quantum result shown in Fig. 3, which converges to a positive value $\Omega_{0}$ in the low- $T$ limit. In this limit, one has for a quantum harmonic approximation:

$$
\left\langle\left|\xi_{j}(\mathbf{k})\right|^{2}\right\rangle_{0}=\frac{\hbar}{2 m \omega_{j}(\mathbf{k})},
$$

and the contribution to $\Omega$ of low-frequency LA and TA modes $(\omega \sim k)$ is proportional to $k$. For the flexural ZA band we have $\omega_{\mathrm{ZA}} \approx \sqrt{\kappa} k^{2}$, so that $k^{2}\left\langle\left|\xi_{j}(\mathbf{k})\right|^{2}\right.$ is independent of $k$ and the sum in Eq. (6) converges to a finite value.

\section{THERMAL EXPANSION}

In the limit $T \rightarrow 0$, the areas $A$ and $A_{p}$ converge to 2.6438 and $2.6388 \AA^{2}$ /atom, respectively. For the classical minimumenergy bilayer one has a value of $2.6169 \AA^{2}$ /atom for both $A$ and $A_{p}$. Then, there is a zero-point expansion of about $1 \%$ associated to an increase in the mean bond length, caused by quantum zero-point vibrations (see above). The difference between in-plane and real area (a $0.2 \%)$ is due to out-of-plane zero-point motion, so that even at $T=0$ the graphene layers are not totally planar, as indicated above.

Associated to the area $A_{p}$, we define an in-plane thermal expansion coefficient (TEC) as

$$
\alpha_{x y}=\frac{1}{A_{p}}\left(\frac{\partial A_{p}}{\partial T}\right)_{P_{x y}} .
$$

In Fig. 4, we show $\alpha_{x y}$ derived from our PIMD simulations for bilayer graphene (solid circles). These data points were obtained from a numerical derivative of the area $A_{p}$ found in the simulations. For comparison, we also display results for graphite (diamonds). In both cases, the dashed lines represent polynomial fits to the data points. The solid line indicates a fit to results of PIMD simulations of monolayer graphene presented in Ref. [28]. All these results correspond to a system size $N=960$. In the three cases, $\alpha_{x y}$ vanishes in the low-temperature limit, in line with the third law of thermodynamics.

The general trend of $\alpha_{x y}$ versus $T$ is similar in the three cases shown in Fig. 4: at low temperatures, $\alpha_{x y}$ decreases

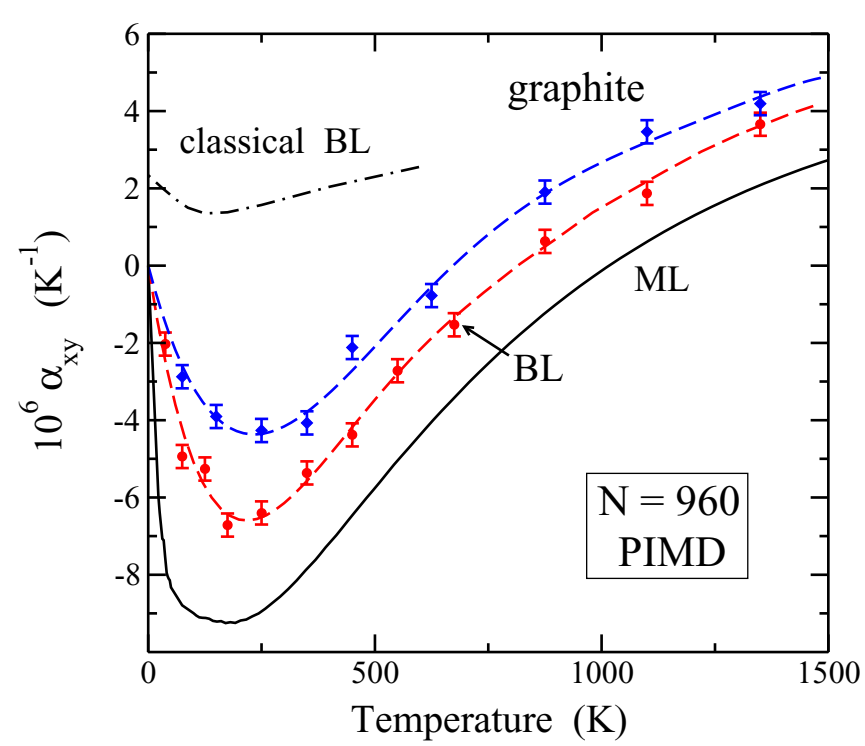

FIG. 4. In-plane thermal expansion coefficients $\alpha_{x y}$ vs temperature as derived from PIMD simulations for cell size $N=960$. Symbols are data points obtained from numerical derivatives of $A_{p}$ for graphene bilayer (BL, circles) and graphite (diamonds). Dashed lines are polynomial fits to the data points. The solid line is a fit to earlier results for a graphene monolayer (ML) [28]. The dashed-dotted line indicates the classical result for the bilayer for temperature up to $600 \mathrm{~K}$.

for rising $T$ and reaches a minimum at a temperature $T_{m}^{\prime}$. The main difference between them appears in the magnitude of the minimum of the curves. Moreover, $T_{m}^{\prime}$ increases from a value of $180 \mathrm{~K}$ for monolayer graphene to $235 \mathrm{~K}$ for graphite. At low temperatures, $\alpha_{x y}$ decreases fast for increasing $T$, and for the bilayer it attains a minimum amounting to $-6.6 \times 10^{-6} \mathrm{~K}^{-1}$ at $T_{m}^{\prime} \approx 220 \mathrm{~K}$. At higher $T, \alpha_{x y}$ approaches zero and becomes positive at $T_{m}=820 \mathrm{~K}$ (where $A_{p}$ takes its minimum value, see Fig. 2). At $T>500 \mathrm{~K}$, the three materials present almost the same dependence of $\alpha_{x y}$ on $T$, apart from rigid shifts of the corresponding curves. Our results for $\alpha_{x y}$ presented in Fig. 4 are qualitatively similar to those derived earlier for monolayer graphene from other theoretical techniques $[65,66]$ and experimental methods $[67,68]$.

For graphite, experimental data of the area TEC $\alpha_{x y}$ display a minimum at a temperature between 200 and $300 \mathrm{~K}$, similar to that derived from our simulations [69-71]. Various data present a minimum of $\approx-3 \times 10^{-6} \mathrm{~K}^{-1}$, somewhat smaller than our result for graphite shown in Fig. 4.

The behavior of the in-plane TEC as a function of temperature can be understood as due to two opposing contributions. First, there appears a trend of the $\mathrm{C}-\mathrm{C}$ distance to grow as $T$ is raised, thus favoring an increase in $A_{p}$. Second, bending of the graphene sheets causes a reduction of its projection on the $(x, y)$ plane, i.e., the in-pane area $A_{p}$. At low $T$, the rise of the in-plane area caused by the first contribution (bond expansion) is overshadowed by the second one (bending), and $d A_{p} / d T<0$. At high $T$, the increase in $\mathrm{C}-\mathrm{C}$ distance dominates the reduction in $A_{p}$ due to out-of-plane atomic displacements, so that one has $d A_{p} / d T>0$. The increase in $T_{m}$ for rising system size shown in Fig. 2 is a consequence of 


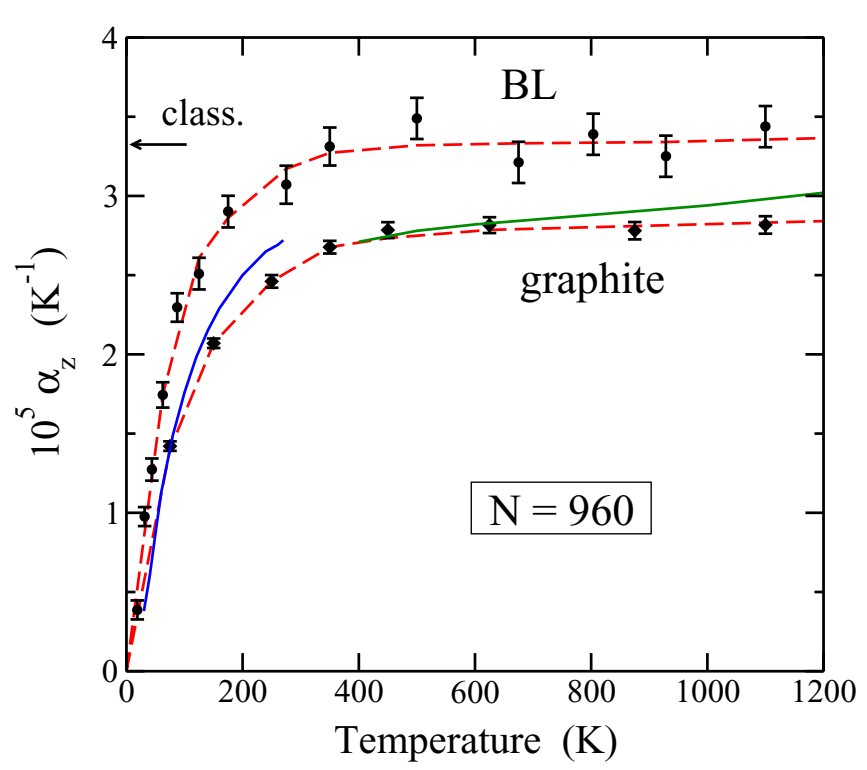

FIG. 5. Thermal expansion coefficient $\alpha_{z}$ in the out-of-plane direction vs the temperature as derived from PIMD simulations for $N=960$. Symbols are data points obtained from temperature derivatives of of the interlayer spacing for bilayer graphene (BL, circles) and graphite (diamonds). Dashed lines are guides to the eye. The blue solid line represents data obtained for graphite by Bailey and Yates [73] from interferometric measurements at $T<300 \mathrm{~K}$. The green solid line is a fit to experimental data of graphite for $T>300 \mathrm{~K}$, presented by Marsden et al. [71]. A horizontal arrow indicates the low-temperature limit of the classical simulations for the bilayer (labeled as "class").

the growth of the out-of-plane bending of the graphene sheets for larger $N$.

One can equally define a TEC $\alpha=(\partial A / \partial T) / A$ for the real area of the graphene sheets. The real area behaves as a function of $T$ in an analogous way to the crystal volume of most 3D solids [72], i.e., it increases at all finite temperatures. The area TEC $\alpha$ is insensitive to the system size [42] and coincides within error bars for monolayer, bilayer graphene, and graphite.

In the low-temperature limit our PIMD simulations yield for bilayer graphene an interlayer spacing, $c$, of $3.3520 \AA$, to be compared with that corresponding to the classical minimum: $c_{0}=3.3372 \AA$ (planar graphene sheets in $\mathrm{AB}$ stacking). This means a zero-point expansion of $1.5 \times 10^{-2} \AA$, i.e., the mean spacing between layers increases by a $0.5 \%$ with respect to the classical prediction. At $T=300 \mathrm{~K}$, PIMD simulations give $c=3.3758 \AA$, and the difference between classical and quantum results is about five times less than in the lowtemperature limit [42].

From the mean interlayer spacing we define the out-ofplane TEC $\alpha_{z}$ as

$$
\alpha_{z}=\frac{1}{c}\left(\frac{\partial c}{\partial T}\right)_{P_{x y}} .
$$

This TEC has been usually called $\alpha_{c}$ in the graphite literature, but we will call it here $\alpha_{z}$ for consistency of our notation. In Fig. 5, we show results for $\alpha_{z}$ derived from our PIMD simulations for bilayer graphene (solid circles) and graphite (diamonds). Dashed lines are guides to the eye. $\alpha_{z}$ turns out to be higher for the bilayer than for graphite at all finite temperatures, since the graphene layers are more free to move in the out-of-plane direction in the bilayer, as compared to graphite. In both cases one observes a fast increase in $\alpha_{z}$ up to about $200 \mathrm{~K}$, which becomes rather slow for $T>400 \mathrm{~K}$.

A blue solid line in Fig. 5 represents $\alpha_{z}$ data obtained for pyrolytic graphite by Bailey and Yates [73] from interferometric measurements at low temperatures. A green solid line represents a fit to experimental data for graphite at $T>300 \mathrm{~K}$ [71]. Both lines fitted to experimental results do not match well one with the other, mainly due to data dispersion in different source references. At high temperature, one observes that $\alpha_{z}$ derived from our PIMD simulations increases slower than the experimental data.

To end this section, we comment on the fact that classical atomistic simulations cannot give reliable results for several properties of graphene (condensed matter in general) at temperatures below the Debye temperature of the material, $\Theta_{D}$ [72,74]. This is the case of thermal expansion coefficients, which have to vanish in the low-temperature limit, according to the third law of thermodynamics [62]. In our case of graphene bilayers, classical simulations yield unphysical finite (positive) values for $\alpha_{x y}$ and $\alpha_{z}$ when $T \rightarrow 0$, as indicated in Figs. 4 and 5 by a dashed-dotted line and an arrow, respectively. This failure of classical simulations is the same as that known for solids when atomic vibrations are described by classical models [72,74] and has been observed earlier for monolayer graphene [27].

In classical simulations, the vibrational states display a (nonrealistic) continuous energy distribution that causes physical anomalies at low temperatures. This is related to the quantization of vibrational states, which is adequately described by path-integral simulations. Since the actual values of $\alpha_{x y}$ and $\alpha_{z}$ are given by the relative population of the excited vibrational states, both variables converge to zero for $T \rightarrow 0$ due to the presence of the energy gap for the vibrational modes. The failure of classical simulations is remedied at relatively high $T$ (the scale is set by $\Theta_{D}$ ), when excited states are appreciably populated. We finally note that simultaneous anomalies at low temperature in thermal expansion coefficients and the specific heat $c_{p}$ derived from classical simulations are expected from the thermodynamic relations between these variables $[28,62,72]$.

\section{COMPRESSIBILITY}

\section{A. In-plane compressibility}

PIMD simulations allow one to obtain insight into the elastic properties of materials under different conditions, i.e., various kinds of external stresses such as hydrostatic or uniaxial. For a two-dimensional material, we understand a hydrostatic stress in a similar way to three-dimensional materials, but applied in a plane (with units of force per unit length). In the language of elasticity this means in our case $\sigma_{x x}=\sigma_{y y}=P_{x y}$ (see Ref. [75]). Then, we define the in-plane isothermal compressibility per layer as

$$
\chi_{x y}=-\frac{n}{A_{p}}\left(\frac{\partial A_{p}}{\partial P_{x y}}\right)_{T} .
$$




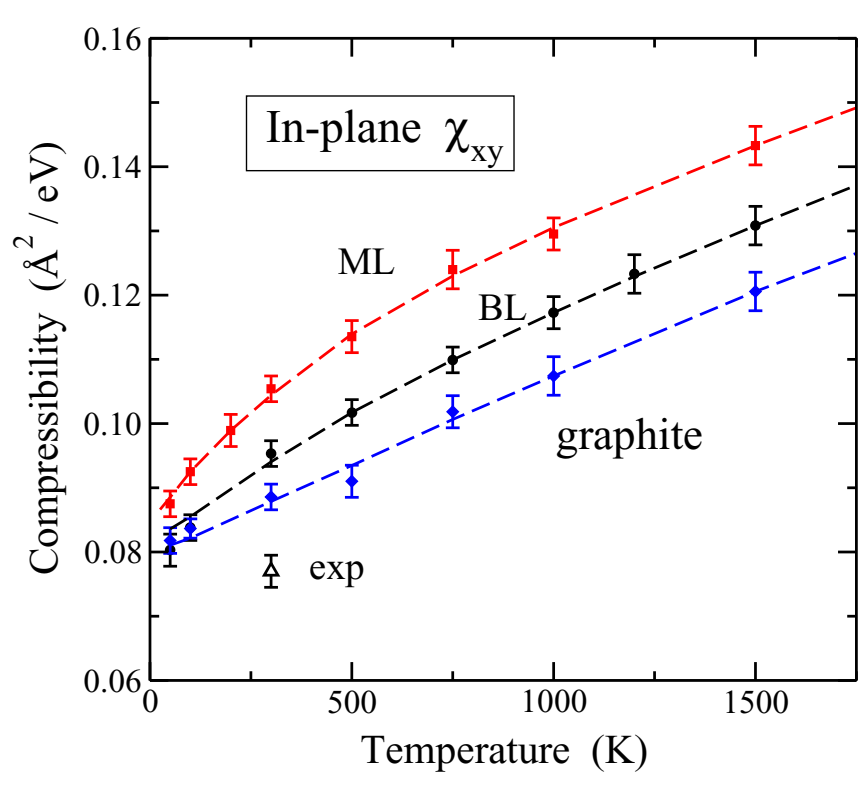

FIG. 6. Temperature dependence of the compressibility $\chi_{x y}$ as derived from PIMD simulations for monolayer (ML, squares), bilayer graphene (BL, circles) and graphite (diamonds). Lines are guides to the eye. An open triangle (labeled "exp") indicates the result derived from experimental data for graphite [78].

where $n$ is the number of layers, i.e., $n=1$ for the monolayer and $n=2$ for bilayer graphene. In this equation, the variables on the right-hand side (r.h.s.) correspond to in-plane quantities, since the pressure $P_{x y}$ in the isothermal-isobaric ensemble employed here is the conjugate variable to the in-plane area $A_{p}$. Note that the normalizing factor $n$ appears in the numerator in Eq. (11), because the inverse of the compressibility (the 2D modulus of hydrostatic compression [75]) is an extensive magnitude proportional to the number of layers.

An alternative way to calculate the compressibility $\chi_{x y}$ is based on the fluctuation formula $[41,76,77]$

$$
\chi_{x y}=\frac{n N \Delta_{p}^{2}}{k_{B} T A_{p}}
$$

where $\Delta_{p}^{2}$ are the mean-square fluctuations of the area $A_{p}$, which in our case are obtained from PIMD simulations at $P_{x y}=0$. In our context, this formula turns out to be more convenient than calculating $\left(\partial A_{p} / \partial P_{x y}\right)_{T}$, because obtaining this derivative by numerical procedures involves additional simulations at nonzero stresses. For some selected temperatures, we have checked that both methods yield the same results for $\chi_{x y}$, inside the statistical error bars.

In Fig. 6, we show the temperature dependence of the compressibility $\chi_{x y}$ of bilayer graphene (solid circles), as derived from our PIMD simulations by using Eq. (12). For comparison, we also present results for monolayer graphene (squares), as well as for graphite (diamonds). At low $T$, we find in the three cases compressibility values close to $\chi_{x y}=0.08 \AA^{2} / \mathrm{eV}$, and the difference between them becomes larger as temperature is raised. $\chi_{x y}$ for bilayer graphene is intermediate between those of monolayer graphene and graphite. Interactions between layers cause a reduction in the out-of-plane vibrational amplitudes of the carbon atoms, so that the layers effectively become "harder," i.e., the in-plane compressibility decreases. Something similar is observed for the out-of-plane compressibility $\chi_{z}$, as shown below.

As noted above, the inverse of $\chi_{x y}, B_{x y}=1 / \chi_{x y}$, is the $2 \mathrm{D}$ modulus of hydrostatic compression [75], with units of eV/ $\AA^{2}$ or $\mathrm{N} / \mathrm{m}$. For graphene, with in-plane hexagonal symmetry, $B_{x y}$ may be written as a function of the elastic constants of the material as

$$
B_{x y}=\frac{1}{2}\left(c_{11}+c_{12}\right),
$$

These elastic constants are related with the Lamé parameters, $\mu$ and $\lambda$ by $c_{11}=\lambda+2 \mu$ and $c_{12}=\lambda$, so that $B_{x y}=\lambda+\mu$ [75].

In the case of graphite, we can make connection of the results obtained here for $\chi_{x y}$ with material properties derived from experiment. For this purpose, we can convert the elastic constants of graphite $C_{11}$ and $C_{12}$ (units of force per square length) into in-plane elastic constants $c_{i j}$ as $c_{i j}=c C_{i j}$, using the mean interlayer distance $c$. Then, for graphite we take $C_{11}=1060 \pm 20 \mathrm{GPa}, C_{12}=180 \pm 20 \mathrm{GPa}$ [78], and $c=3.3538 \AA$ [79], and find $\chi_{x y}=1 / B_{x y}=0.077(2) \AA^{2} / \mathrm{eV}$. For comparison with the results of our simulations, this data point is shown in Fig. 6 as an open triangle at $300 \mathrm{~K}$. The result of our simulations for graphite at $T=300 \mathrm{~K}$ is somewhat higher than that derived from experimental data.

\section{B. Out-of-plane compressibility}

We now turn to the compressibility $\chi_{z}$ of bilayer graphene in the out-of-plane direction. Similarly to the in-plane compressibility $\chi_{x y}, \chi_{z}$ can be calculated from the interlayer spacing and its fluctuations along a simulation run at a given temperature. The isothermal compressibility in the $z$ direction is defined as

$$
\chi_{z}=-\frac{1}{V} \frac{\partial V}{\partial P_{z}},
$$

where $V=c L_{x} L_{y}$ and $P_{z}$ is a uniaxial stress in the out-of-plane direction. The compressibility $\chi_{z}$ of bilayer graphene at a temperature $T$ may be calculated from PIMD simulations with $P_{z}=0$ by employing the fluctuation formula $[76,80]$

$$
\chi_{z}=\frac{\Delta_{V}^{2}}{k_{B} T V},
$$

where the volume mean-square fluctuations associated to changes in the interlayer distance $c$ are given by $\Delta_{V}^{2}=$ $L_{x}^{2} L_{y}^{2} \Delta_{c}^{2}$. Then, we obtain $\chi_{z}$ by using the expression

$$
\chi_{z}=\frac{L_{x} L_{y}}{k_{B} T} \frac{\Delta_{c}^{2}}{c} .
$$

Note that in this expression $L_{x}, L_{y}$, and $c$ indicate mean values of these variables along a simulation run at temperature $T$.

The temperature dependence of $\chi_{z}$ is shown in Fig. 7. Solid circles are data points obtained from our PIMD simulations for bilayer graphene. Besides, we display in Fig. 7 data for the compressibility of graphite, derived also from PIMD simulations, using Eq. (16). Both sets of results converge at low temperature to the same value of the compressibility (within error bars): $\chi_{z}=2.79(2) \times 10^{-2} \mathrm{GPa}^{-1}$, because the MSDs $\Delta_{c}^{2}$ are found to be nearly identical for bilayer graphene 


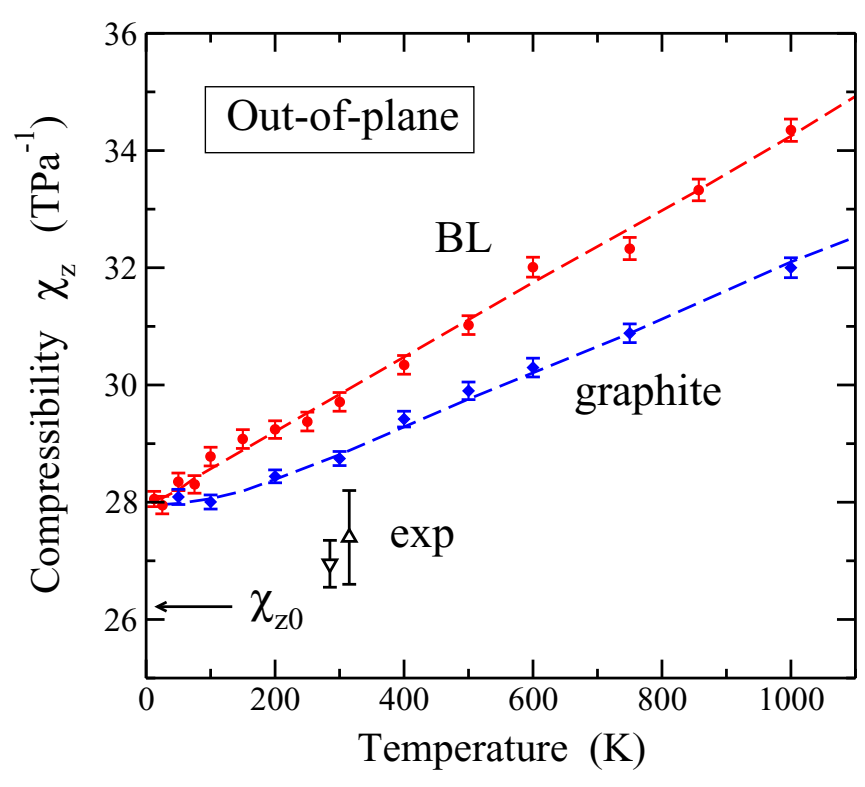

FIG. 7. Temperature dependence of the compressibility $\chi_{z}$ as derived from PIMD simulations for bilayer graphene (BL, circles) and graphite (diamonds). Lines are guides to the eye. Open triangles indicate results derived from experimental data of graphite at room temperature: triangle up from Ref. [78] and triangle down from Ref. [81]. A horizontal arrow shows the classical zero-temperature limit $\chi_{z 0}$.

and graphite. For higher $T, \Delta_{c}^{2}$ is smaller for graphite, and therefore its compressibility $\chi_{z}$ is lower than that of bilayer graphene.

We note that the classical compressibility $\chi_{z 0}$ for $T \rightarrow$ 0 can be calculated from the dependence of the system energy on the interlayer spacing $c$ close to the minimumenergy value $c_{0}$. This yields $\chi_{z 0}=2.63 \times 10^{-12} \mathrm{~cm}^{2} \mathrm{dyn}^{-1}$ or $0.0263 \mathrm{GPa}^{-1}$ (see Ref. [42]), a value indicated in Fig. 7 by a horizontal arrow. This means an appreciable increase of a $6 \%$ in the low-temperature quantum value of $\chi_{z}$ with respect to the classical limit.

The compressibility $\chi_{z}$ coincides in the case of graphite with the elastic compliance constant $S_{33}$ of this material, since this constant connects stress and strain in the $z$ direction [71]. In Fig. 7, we show $S_{33}$ obtained for pyrolytic graphite from neutron diffraction data combined with a force model [81] (triangle down), and from ultrasonic test methods [78] (triangle up). These data were obtained at room temperature and are horizontally moved around $300 \mathrm{~K}$ in Fig. 7 for the sake of clarity. Note that for graphite $S_{33}$ is related to the elastic constant $C_{33}$ as $S_{33} C_{33} \approx 1$, and the difference between $S_{33}$ and $C_{33}^{-1}$ is less than the error bars of the experimental data $[78,81]$. Our results overestimate the compressibility $\chi_{z}$ of graphite by nearly a $5 \%$ with respect to those data derived from experiments at room temperature.

Komatsu [82] found at low-temperature $(T \approx 2 \mathrm{~K})$ a value of the elastic constant $C_{33}=35.6 \mathrm{GPa}$ from specific-heat measurements of natural and pile graphite, which translates to $\chi_{z}=0.0282 \mathrm{GPa}^{-1}$. This value (not shown in Fig. 7) is close to our results for graphite, but there is no available error bar for it.

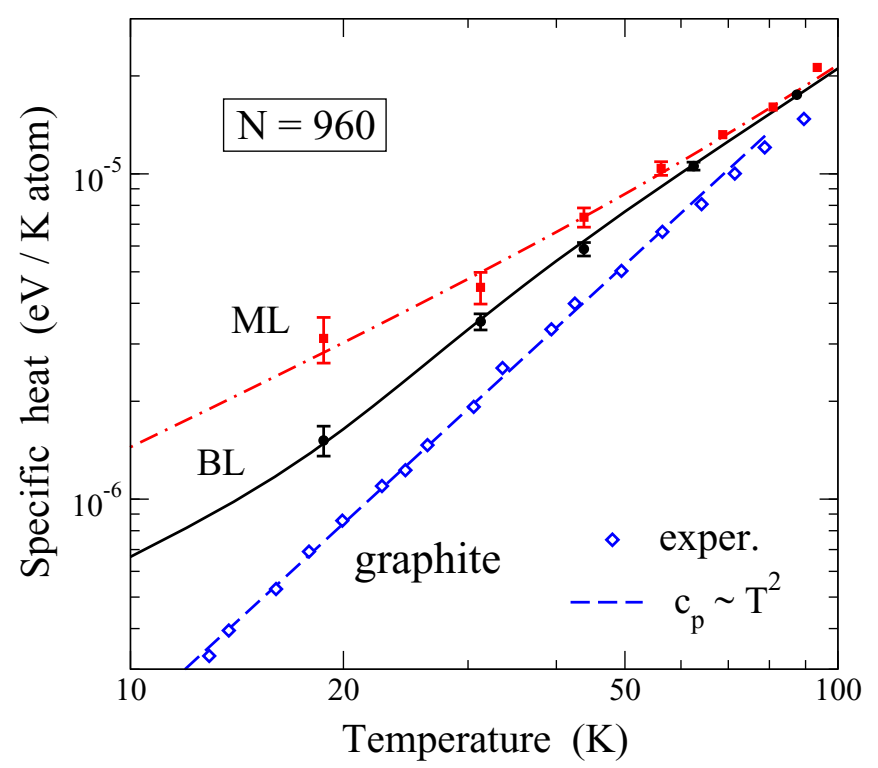

FIG. 8. Specific heat of graphene as a function of temperature. Solid symbols represent results for $c_{p}$ derived from PIMD simulations for $N=960$ : squares for graphene monolayer and circles for the bilayer. The solid line is $c_{v}$ obtained from the 12 phonon bands of bilayer graphene, corresponding to the LCBOPII potential in a harmonic approximation. The dashed-dotted line indicates $c_{v}$ for monolayer graphene in the HA [28]. Open diamonds represent experimental data for graphite obtained by Desorbo and Tyler [88]. The dashed line shows the dependence $c_{p} \propto T^{2}$.

\section{SPECIFIC HEAT}

The calculation of low-temperature specific heats of materials by means of path-integral simulations is not straightforward in general. Even obtaining the Debye law $c_{p} \sim T^{3}$ for 3D solids has been a challenge for PIMD, because of the effective low-frequency cut-off associated to the finite size of the simulation cells $[83,84]$. This situation is improved in simulations of 2D materials such as graphene, mainly for two reasons. First, the length of the cell sides scales as $L \sim$ $N^{1 / d}$ ( $d$, dimension of the space), and the minimum wave number $k_{0}$ available in the simulation scales as $k_{0} \sim N^{-1 / d}$. Thus, for increasing number of atoms, $k_{0}$ decreases faster for $d=2$ than for $d=3$. This means that the low-frequency region is described better for $2 \mathrm{D}$ materials, and therefore also the low-temperature region. Second, the internal energy for graphene rises at low temperature as $T^{2}$ (i.e., $c_{p} \sim T$ ), which is a fast increase at low temperature, when compared with the typical expectancy $\left(E \sim T^{4}\right)$ for the phonon contribution in 3D materials $\left(c_{p} \sim T^{3}\right)$.

The specific heat of graphene is controlled by the vibrational contribution, the electronic part $c_{p}^{\mathrm{el}}$ being negligible with respect to the former. In fact, $c_{p}^{\text {el }}$ has been estimated in various works, and it results to be between three and four orders of magnitude less than the vibrational part [85-87].

In Fig. 8, we present the temperature dependence of the specific heat in the low-temperature region in a logarithmic plot. Solid symbols are results for $c_{p}$ obtained from PIMD simulations for $N=960$ : circles for a graphene bilayer and squares for a monolayer. They were obtained from a 
numerical derivative of the internal energy $E(T)$. The solid line represents $c_{v}$ for the bilayer, derived from the harmonic approximation given by Eq. (2) for the same cell size. The dashed-dotted line indicates the HA for the monolayer, obtained by adding the contributions of the six phonon bands appearing in this case [28].

We first observe in Fig. 8 a good agreement between the results of the HA and those derived from PIMD simulations at $T<100 \mathrm{~K}$ in both cases, monolayer and bilayer graphene. Results of the simulations are close to the HA up to about $300 \mathrm{~K}$, and at higher $T$ they gradually depart from the solid line, in a temperature region where anharmonic effects are expected to be observable. For monolayer graphene, one observes a linear dependence of the specific heat for $T \lesssim$ $40 \mathrm{~K}$ (slope unity in the logarithmic plot), given by $c_{p} \approx C T$ with $C=1.4 \times 10^{-7} \mathrm{eV} \mathrm{K}^{-2}$ For the bilayer, a similar trend with a linear dependence of $c_{p}$ appears also at low $T$ with a constant $C=5.0 \times 10^{-8} \mathrm{eV} \mathrm{K}^{-2}$, but for $T \gtrsim 15 \mathrm{~K}$ the temperature dependence becomes superlinear. This trend is explained below.

For comparison with the results of our simulations, we also show in Fig. 8 experimental data for $c_{p}$ of graphite, obtained by Desorbo and Tyler from calorimetric measurements [88] (open diamonds). The specific heat of graphite has been thoroughly analyzed in a wide range of temperatures [81,89-91]. For this 3D material, $c_{p}$ increases as $T^{3}$ for $T<10 \mathrm{~K}$ (a region not reached in our simulations and not presented in Fig. 8). For $T$ between 10 and $100 \mathrm{~K}, c_{p}$ rises as $T^{2}$, a typical dependence in strongly anisotropic solids $[81,86]$. The most important difference between graphite and graphene (monolayer and bilayer) in this temperature range consists in the dominant contribution to $c_{p}$ coming from phonons with linear dispersion relation $(\omega \sim k)$ for small $k$ in graphite. At room temperature $(T=300 \mathrm{~K})$ the measured specific heat of graphite equals $8.90 \times 10^{-5} \mathrm{eV} /(\mathrm{K}$ atom $)$, or $8.59 \mathrm{~J} \mathrm{~K}^{-1} \mathrm{~mol}^{-1}$ [88], to be compared with the result of our PIMD simulations for bilayer graphene, $c_{p}=9.2( \pm 0.1) \times$ $10^{-5} \mathrm{eV} \mathrm{K}^{-1}$ atom $^{-1}$, and for a monolayer, $c_{p}=9.4( \pm 0.1) \times$ $10^{-5} \mathrm{eV} \mathrm{K}^{-1}$ atom $^{-1}$ [28].

One can also calculate the specific heat $c_{v}$ from constant $-A_{p}$ simulations, analogous to $N V T$ simulations in $3 \mathrm{D}$ materials. From thermodynamic considerations, one should have $c_{v} \leqslant$ $c_{p}$ at any temperature, but the difference between them for bilayer graphene is smaller than the statistical error bar of our numerical results, and they are indistinguishable in the results derived from PIMD simulations [28].

The difference between $c_{p}$ and $c_{v}$ can be obtained from the formula [28]

$$
c_{p}-c_{v}=\frac{n T \alpha_{x y}^{2} A_{p}}{\chi_{x y}}
$$

which is similar to the well-known thermodynamic expression for this difference of specific heats in 3D systems $[62,76]$. The variables present on the r.h.s. of Eq. (17) refer to in-plane properties, since the pressure appearing in our isothermalisobaric ensemble is the conjugate variable of the in-plane area $A_{p}$.

For thermodynamic consistency, one needs $c_{p} \geqslant c_{v}$, in accord with Eq. (17), and we have $c_{p}-c_{v}=0$ whenever $\alpha_{x y}$

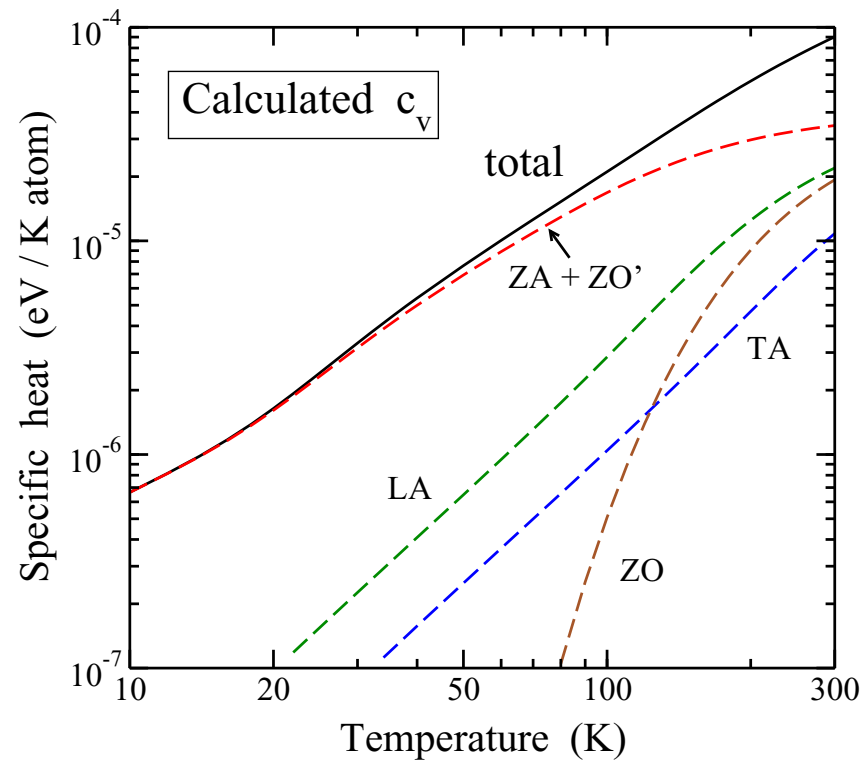

FIG. 9. Contributions $c_{v}^{j}$ of the phonon bands to the specific heat of bilayer graphene as a function of temperature.

vanishes. This happens for bilayer graphene at $T_{m} \approx 850 \mathrm{~K}$, as shown in Sec. III (apart from the trivial coincidence $c_{p}=$ $c_{v}=0$ at $T=0$ ). In the interval from $T=0$ to $850 \mathrm{~K}$, the maximum difference is reached at $T \approx 200 \mathrm{~K}$, close to the maximum of $\left|\alpha_{x y}\right|$, where we find using Eq. (17): $c_{p}-c_{v}=$ $5.6 \times 10^{-7} \mathrm{eV} \mathrm{K}^{-1}$ atom $^{-1}$. For $T<50 \mathrm{~K}$, we have $c_{p}-$ $c_{v}<4 \times 10^{-8} \mathrm{eV} \mathrm{K}^{-1}$ atom $^{-1}$, less than the statistical error bars of the results for $c_{p}$ derived from our PIMD simulations.

The low-temperature behavior of the specific heat can be analyzed by considering a continuous model for frequencies and wave numbers, as in the well-known Debye model for solids [72] (see Appendix B). At low $T, c_{v}$ is controlled by the input of acoustic modes with small $k$. In the case of graphene, these are TA and LA modes with $\omega_{j} \propto k$ and ZA modes with $\omega_{j} \propto k^{2}$. Note that an effective stress $\sigma$ introduces a linear contribution for ZA modes of small $k$, but this will be negligible for the temperatures considered here and vanishing external in-plane stress. For bilayer graphene, it is appreciable at $T>15 \mathrm{~K}$ the role of the layer-breathing ZO' band, which is nearly flat close to the $\Gamma$ point $(k=0)$, with a frequency $\omega_{0}=92 \mathrm{~cm}^{-1}[42,55]$.

To understand the behavior of the specific heat of bilayer graphene in the temperature region displayed in Fig. 8, we discuss the contributions of the different phonon branches in the HA. In Fig. 9, we present as dashed lines these contributions as a function of $T$. At temperatures lower than $10 \mathrm{~K}$, the specific heat is controlled by the flexural ZA modes with $\omega(k) \sim k^{2}$ and out-of-plane displacements. This gives $c_{v}^{j} \sim T$, as in the case of monolayer graphene (see Appendix B). For the bilayer, however, the input of the ZO' band is relevant for $T \gtrsim 15 \mathrm{~K}$, and $c_{v}$ appreciably departs from linearity. The contributions of the acoustic LA and TA branches $(\omega \sim k)$ appear in Fig. 9 for $T>20 \mathrm{~K}$ with a slope of two, i.e., $c_{v}^{j} \sim$ $T^{2}$. The input of the optical ZO band increases exponentially at low $T$ and becomes observable at temperatures in the order 
of $100 \mathrm{~K}$. The other optical bands (LO and TO), with higher frequencies, are important for the specific heat at $T>300 \mathrm{~K}$.

The specific heat of graphene bilayer is lower than that of the monolayer due to the relative contributions of acoustic phonons at low $T$. In particular, the shape of the flexural ZA band is nearly the same in both cases, and it is not degenerate. This means that it contributes less in bilayer graphene (12 bands) than in the monolayer (6 bands).

We finally note that several atomistic simulations of graphene monolayers and bilayers have been carried out in the past using classical Monte Carlo and molecular dynamics simulations. These are well-established methods to study structural, dynamical, and thermodynamic properties in condensed matter, but some of these properties may be far from the corresponding real values at temperatures lower than the Debye temperature $\Theta_{D}$ of the considered material [72], as indicated for the thermal expansion in Sec. IV. Thus values presented in the literature for the specific heat of graphene monolayers [92] and bilayers [39], derived from classical simulations, are close to the Dulong-Petit specific heat, i.e., $c_{v}^{\mathrm{cl}}=3 k_{B}$. This means in our units $c_{v}^{\mathrm{cl}}=2.6 \times 10^{-4} \mathrm{eV} \mathrm{K}^{-1}$ atom $^{-1}$, which turns out to be about three times larger than the value obtained from our quantum PIMD simulations at $300 \mathrm{~K}$. The difference between classical and quantum results increases as temperature is lowered, and at $T=20 \mathrm{~K}$ the classical value is two orders of magnitude larger than the quantum result. Even at $T=1000 \mathrm{~K}$ the quantum data are still appreciably lower than the classical limit.

\section{SUMMARY}

PIMD simulations have revealed as a suitable tool to study thermodynamic properties of graphene bilayers. In this paper, we have presented results obtained in the isothermal-isobaric ensemble in a wide range of temperatures and zero external stress. We have concentrated on physical properties as the excess area, thermal expansion, in-plane and out-of-plane compressibility, and specific heat. Explicit consideration of the quantum character of atomic nuclei is crucial for a realistic description of these crystalline membranes, even for $T$ higher than room temperature. This is particularly important for the heat capacity and compressibility.

A thermal contraction of the in-plane area $A_{p}$ appears in bilayer graphene in a similar way to an isolated monolayer, although this contraction is less important in the former case. This is due to a reduction of out-of-plane vibrational amplitudes of the $\mathrm{C}$ atoms in the bilayer, associated to interlayer interactions. We find a negative $\alpha_{x y}$ for $T \lesssim 800 \mathrm{~K}$, and it becomes positive at higher temperature. The difference $A-A_{p}$ between the real area $A$ and the in-plane area $A_{p}$ grows as temperature rises and deviations from planarity of the graphene sheets become more appreciable. This has been quantified by the dimensionless excess area $\Omega$, which converges to a value $\Omega_{0}=2 \times 10^{-3}$ for $T \rightarrow 0$, due to quantum zero-point motion.

The in-plane $\chi_{x y}$ and out-of-plane $\chi_{z}$ compressibilities of graphene bilayers have been obtained from the fluctuations of the in-plane area and the interlayer distance, respectively. This procedure accurately yields the increase in $\chi_{x y}$ and $\chi_{z}$ as $T$ is raised.
Comparison of our simulation results with those yielded by a HA for the vibrational modes has allowed us to assess the effects of anharmonicity in finite-temperature properties of graphene bilayers. Such anharmonicity clearly shows up at temperatures higher than $200 \mathrm{~K}$, as shown in Fig. 3 for the excess area. At lower temperatures, however, thermal properties of the graphene bilayers considered here are well described by the HA, using the vibrational frequencies obtained for the classical equilibrium geometry at $T=0$.

At the lowest temperatures studied here $(T>10 \mathrm{~K})$, the HA predicts a linear dependence of the specific heat $c_{v}=C T$, with $C=5.0 \times 10^{-8} \mathrm{eV} \mathrm{K}^{-2}$, and for $T \gtrsim 15 \mathrm{~K}$ the temperature dependence becomes superlinear, in agreement with the results of our PIMD simulations. This trend is different than that corresponding to monolayer graphene, due to the contribution of the layer-breathing $\mathrm{ZO}^{\prime}$ band in the case of the bilayer.

PIMD simulations similar to those presented here can provide insight about the thermal properties of free-standing graphene multilayers under tensile and compressive stress. This would give information on the relative stability of these multilayers in a stress-temperature phase diagram.

\section{ACKNOWLEDGMENTS}

The authors acknowledge the help of J. H. Los in the implementation of the LCBOPII potential. This work was supported by Ministerio de Ciencia, Innovación y Universidades (Spain) through Grants No. FIS2015-64222-C2 and No. PGC2018-096955-B-C44.

\section{APPENDIX A: CALCULATION OF THE EXCESS AREA}

In the continuum limit, the instantaneous real area $A_{\text {inst }}$ of a graphene sheet is given by $[41,58,59]$

$$
A_{\text {inst }}=\int_{A_{p}} d x d y \sqrt{1+|\nabla h(\mathbf{r})|^{2}},
$$

where $\mathbf{r} \equiv(x, y)$ indicates the $2 \mathrm{D}$ position and $h(\mathbf{r})$ is the height of the surface, i.e., the distance to the mean $(x, y)$ plane of the sheet. For small $|\nabla h(\mathbf{r})|$ [in fact for $(\partial h / \partial x)^{2}+$ $(\partial h / \partial y)^{2} \ll 1$, which is the case here], one has

$$
A_{\mathrm{inst}} \approx \int_{A_{p}} d x d y\left[1+\frac{1}{2}|\nabla h(\mathbf{r})|^{2}\right] .
$$

We now write the out-of-plane displacement $h(\mathbf{r})$ as a Fourier series

$$
h(\mathbf{r})=\frac{1}{\sqrt{N}} \sum_{\mathbf{k}} \mathrm{e}^{i \mathbf{k} \cdot \mathbf{r}} H(\mathbf{k})
$$

with wave vectors $\mathbf{k}=\left(k_{x}, k_{y}\right)$ in the $2 \mathrm{D}$ hexagonal Brillouin zone, i.e., $k_{x}=2 \pi n_{x} / L_{x}$ and $k_{y}=2 \pi n_{y} / L_{y}$ with integers $n_{x}$ and $n_{y}$ [43]. The Fourier components are given by

$$
H(\mathbf{k})=\frac{\sqrt{N}}{A_{p}} \int_{A_{p}} d x d y \mathrm{e}^{-i \mathbf{k} \cdot \mathbf{r}} h(\mathbf{r}) .
$$


With $H(\mathbf{k})$ so defined, the thermal average of MSD in the $z$ direction is given by

$$
\left\langle h(\mathbf{r})^{2}\right\rangle=\frac{1}{N} \sum_{\mathbf{k}}\left\langle|H(\mathbf{k})|^{2}\right\rangle .
$$

Thus we have

$$
\nabla h(\mathbf{r})=\frac{i}{\sqrt{N}} \sum_{\mathbf{k}} \mathbf{k} \mathrm{e}^{i \mathbf{k} \cdot \mathbf{r}} H(\mathbf{k})
$$

and

$$
|\nabla h(\mathbf{r})|^{2}=\frac{1}{N} \sum_{\mathbf{k}_{1}, \mathbf{k}_{2}} \mathbf{k}_{1} \cdot \mathbf{k}_{2} \mathrm{e}^{i\left(\mathbf{k}_{1}-\mathbf{k}_{2}\right) \cdot \mathbf{r}} H\left(\mathbf{k}_{1}\right) H\left(\mathbf{k}_{2}\right)^{*},
$$

which yields

$$
\left\langle|\nabla h(\mathbf{r})|^{2}\right\rangle=\frac{1}{N} \sum_{\mathbf{k}} k^{2}\left\langle|H(\mathbf{k})|^{2}\right\rangle .
$$

Then, the mean real area is given by

$$
A=\left\langle A_{\text {inst }}\right\rangle=A_{p}+\frac{A_{p}}{2 N} \sum_{\mathbf{k}} k^{2}\left\langle|H(\mathbf{k})|^{2}\right\rangle,
$$

and for uncoupled vibrational modes in the out-of-plane direction (harmonic approximation), $\left\langle|H(\mathbf{k})|^{2}\right\rangle$ can be written as a sum of their MSDs:

$$
\left\langle|H(\mathbf{k})|^{2}\right\rangle=\sum_{j}\left\langle\left|\xi_{j}(\mathbf{k})\right|^{2}\right\rangle
$$

so that

$$
\Omega=\frac{A-A_{p}}{A_{p}}=\frac{1}{2 N} \sum_{j, \mathbf{k}} k^{2}\left\langle\left|\xi_{j}(\mathbf{k})\right|^{2}\right\rangle
$$

with

$$
\left\langle\left|\xi_{j}(\mathbf{k})\right|^{2}\right\rangle=\frac{\hbar}{2 m \omega_{j}(\mathbf{k})} \operatorname{coth}\left(\frac{1}{2} \beta \hbar \omega_{j}(\mathbf{k})\right) .
$$

The sum in $j$ in Eq. (A11) is extended to the phonon bands with displacements in the $z$ direction (ZA, ZO', and the twofold degenerate $\mathrm{ZO}$ ). Note that in our simulations the in-plane area also fluctuates, but its fluctuations are not considered in the harmonic calculation presented here.

\section{APPENDIX B: PHONON CONTRIBUTIONS TO THE LOW-TEMPERATURE SPECIFIC HEAT}

Here we present a continuous model for wave numbers and frequencies of vibrational modes, to find an analytic dependence for the contributions of the different phonon bands to the low-temperature specific heat of bilayer graphene. For a phonon branch with dispersion relation $\omega_{j} \propto k^{n}$ for small $k$, the low-temperature contribution to the specific heat may be approximated as

$$
c_{v}^{j}(T) \approx \frac{k_{B}}{2} \int_{k_{0}}^{k_{m}} \frac{\left[\frac{1}{2} \beta \hbar \omega_{j}(k)\right]^{2}}{\sinh ^{2}\left[\frac{1}{2} \beta \hbar \omega_{j}(k)\right]} \rho(k) d k,
$$

where $k_{m}$ is the maximum wave number $k_{m}=\left(2 \pi / A_{0}\right)^{1 / 2}, A_{0}$ is the in-plane area for the minimum-energy configuration, and $\rho(k)=A_{0} k / 2 \pi$ for $2 \mathrm{D}$ systems. From the dispersion relation $\omega_{j}(k)$, we have a vibrational density of states

$$
\bar{\rho}_{r}(\omega)=\rho(k) \frac{d k}{d \omega} \sim \omega^{\frac{2}{n}-1}
$$

so that

$$
c_{v}^{j}(T) \sim k_{B} \int_{\omega_{0}}^{\omega_{m}} \frac{\left(\frac{1}{2} \beta \hbar \omega\right)^{2}}{\sinh ^{2}\left(\frac{1}{2} \beta \hbar \omega\right)} \omega^{\frac{2}{n}-1} d \omega .
$$

Taking the limit $\omega_{0} \rightarrow 0(N \rightarrow \infty)$ and putting $x=$ $\frac{1}{2} \beta \hbar \omega$, we have

$$
c_{v}^{j} \sim k_{B} \frac{K}{(\beta \hbar)^{\frac{2}{n}}} \int_{0}^{x_{m}} \frac{x^{\frac{2}{n}+1}}{\sinh ^{2} x} d x,
$$

$K$ being a constant. At low temperature, $k_{B} T \ll \hbar \omega_{m}$ (large $x_{m}$ ), we have $c_{v}^{j} \sim T^{2 / n}$. In general, for $d$-dimensional systems, one has an exponent $d / n[93,94]$. Then, in graphene we expect for the ZA phonon branch $(n=2): c_{v}^{\mathrm{ZA}} \sim T$, and for the acoustic LA and TA branches $(n=1): c_{v}^{\mathrm{ac}} \sim T^{2}$.

For the ZO' band in bilayer graphene, we have $\omega \approx \omega_{0}$ for small $k$. Then, at low $T$, the corresponding contribution to the specific heat, $c_{v}^{Z O^{\prime}}$, coincides with that of a collection of harmonic oscillators with frequency $\omega_{0}$, i.e., $c_{v}^{Z O^{\prime}} \sim$ $\exp \left(-\hbar \omega_{0} / k_{B} T\right)$.
[1] Y. Cao, V. Fatemi, S. Fang, K. Watanabe, T. Taniguchi, E. Kaxiras, and P. Jarillo-Herrero, Nature (London) 556, 43 (2018).

[2] F. Guinea and N. R. Walet, PNAS 115, 13174 (2018).

[3] M. Yankowitz, S. Chen, H. Polshyn, Y. Zhang, K. Watanabe, T. Taniguchi, D. Graf, A. F. Young, and C. R. Dean, Science 363, 1059 (2019).

[4] Y. Cao, V. Fatemi, A. Demir, S. Fang, S. L. Tomarken, J. Y. Luo, J. D. Sanchez-Yamagishi, K. Watanabe, T. Taniguchi, E. Kaxiras et al., Nature (London) 556, 80 (2018).

[5] H. C. Po, L. Zou, A. Vishwanath, and T. Senthil, Phys. Rev. X 8, 031089 (2018).
[6] L. A. Gonzalez-Arraga, J. L. Lado, F. Guinea, and P. San-Jose, Phys. Rev. Lett. 119, 107201 (2017).

[7] A. O. Sboychakov, A. V. Rozhkov, A. L. Rakhmanov, and F. Nori, Phys. Rev. Lett. 120, 266402 (2018).

[8] J. C. Meyer, A. K. Geim, M. I. Katsnelson, K. S. Novoselov, D. Obergfell, S. Roth, C. Girit, and A. Zettl, Solid State Commun. 143, 101 (2007).

[9] M. Gibertini, A. Tomadin, M. Polini, A. Fasolino, and M. I. Katsnelson, Phys. Rev. B 81, 125437 (2010).

[10] S. A. Safran, Statistical Thermodynamics of Surfaces, Interfaces, and Membranes (Addison Wesley, New York, 1994).

[11] D. Nelson, T. Piran, and S. Weinberg, Statistical Mechanics of Membranes and Surfaces (World Scientific, London, 2004). 
[12] E. Chacón, P. Tarazona, and F. Bresme, J. Chem. Phys. 143, 034706 (2015).

[13] T. Ruiz-Herrero, E. Velasco, and M. F. Hagan, J. Phys. Chem. B 116, 9595 (2012).

[14] A. A. Balandin, Nat. Mater. 10, 569 (2011).

[15] A. Alofi and G. P. Srivastava, Phys. Rev. B 87, 115421 (2013).

[16] A. Alofi and G. P. Srivastava, Appl. Phys. Lett. 104, 031903 (2014).

[17] B. Amorim, R. Roldan, E. Cappelluti, A. Fasolino, F. Guinea, and M. I. Katsnelson, Phys. Rev. B 89, 224307 (2014).

[18] S. Mann, P. Rani, R. Kumar, G. S. Dubey, and V. K. Jindal, RSC Adv. 6, 12158 (2016).

[19] O. Coquand and D. Mouhanna, Phys. Rev. E 94, 032125 (2016).

[20] A. Fasolino, J. H. Los, and M. I. Katsnelson, Nat. Mater. 6, 858 (2007).

[21] E. Cadelano, P. L. Palla, S. Giordano, and L. Colombo, Phys. Rev. Lett. 102, 235502 (2009).

[22] E. Akatyeva and T. Dumitrica, J. Chem. Phys. 137, 234702 (2012).

[23] Y. Magnin, G. D. Foerster, F. Rabilloud, F. Calvo, A. Zappelli, and C. Bichara, J. Phys.: Condens. Matter 26, 185401 (2014).

[24] J. H. Los, A. Fasolino, and M. I. Katsnelson, Phys. Rev. Lett. 116, 015901 (2016).

[25] A. Politano, B. Borca, M. Minniti, J. J. Hinarejos, A. L. Vazquez de Parga, D. Farias, and R. Miranda, Phys. Rev. B 84, 035450 (2011).

[26] B. G. A. Brito, L. Cândido, G.-Q. Hai, and F. M. Peeters, Phys. Rev. B 92, 195416 (2015).

[27] C. P. Herrero and R. Ramírez, J. Chem. Phys. 145, 224701 (2016).

[28] C. P. Herrero and R. Ramírez, J. Chem. Phys. 148, 102302 (2018).

[29] J. Hasik, E. Tosatti, and R. Martonak, Phys. Rev. B 97, 140301(R) (2018).

[30] N. Mounet and N. Marzari, Phys. Rev. B 71, 205214 (2005).

[31] T. Shao, B. Wen, R. Melnik, S. Yao, Y. Kawazoe, and Y. Tian, J. Chem. Phys. 137, 194901 (2012).

[32] W. Gao and R. Huang, J. Mech. Phys. Solids 66, 42 (2014).

[33] R. P. Feynman, Statistical Mechanics (Addison-Wesley, New York, 1972).

[34] M. J. Gillan, Philos. Mag. A 58, 257 (1988).

[35] D. M. Ceperley, Rev. Mod. Phys. 67, 279 (1995).

[36] C. P. Herrero and R. Ramírez, J. Phys.: Condens. Matter 26, 233201 (2014).

[37] J. H. Los, L. M. Ghiringhelli, E. J. Meijer, and A. Fasolino, Phys. Rev. B 72, 214102 (2005).

[38] L. M. Ghiringhelli, J. H. Los, A. Fasolino, and E. J. Meijer, Phys. Rev. B 72, 214103 (2005).

[39] K. V. Zakharchenko, J. H. Los, M. I. Katsnelson, and A. Fasolino, Phys. Rev. B 81, 235439 (2010).

[40] K. V. Zakharchenko, M. I. Katsnelson, and A. Fasolino, Phys. Rev. Lett. 102, 046808 (2009).

[41] R. Ramírez and C. P. Herrero, Phys. Rev. B 95, 045423 (2017).

[42] C. P. Herrero and R. Ramírez, J. Chem. Phys. 150, 204707 (2019)

[43] R. Ramírez, E. Chacón, and C. P. Herrero, Phys. Rev. B 93, 235419 (2016).

[44] P. Lambin, Appl. Sci. 4, 282 (2014).
[45] M. E. Tuckerman and A. Hughes, in Classical and Quantum Dynamics in Condensed Phase Simulations, edited by B. J. Berne, G. Ciccotti, and D. F. Coker (Word Scientific, Singapore, 1998), p. 311.

[46] G. J. Martyna, A. Hughes, and M. E. Tuckerman, J. Chem. Phys. 110, 3275 (1999).

[47] M. E. Tuckerman, B. J. Berne, G. J. Martyna, and M. L. Klein, J. Chem. Phys. 99, 2796 (1993).

[48] S. Nosé, J. Chem. Phys. 81, 511 (1984).

[49] W. G. Hoover, Phys. Rev. A 31, 1695 (1985).

[50] G. J. Martyna, M. E. Tuckerman, D. J. Tobias, and M. L. Klein, Mol. Phys. 87, 1117 (1996).

[51] M. F. Herman, E. J. Bruskin, and B. J. Berne, J. Chem. Phys. 76, 5150 (1982).

[52] C. P. Herrero, R. Ramírez, and E. R. Hernández, Phys. Rev. B 73, 245211 (2006).

[53] C. P. Herrero and R. Ramírez, J. Chem. Phys. 134, 094510 (2011).

[54] L. J. Karssemeijer and A. Fasolino, Surf. Sci. 605, 1611 (2011).

[55] J.-A. Yan, W. Y. Ruan, and M. Y. Chou, Phys. Rev. B 77, 125401 (2008).

[56] A. K. Singh and R. G. Hennig, Phys. Rev. B 87, 094112 (2013).

[57] E. N. Koukaras, G. Kalosakas, C. Galiotis, and K. Papagelis, Sci. Rep. 5, 12923 (2015).

[58] A. Imparato, J. Chem. Phys. 124, 154714 (2006).

[59] Q. Waheed and O. Edholm, Biophys. J. 97, 2754 (2009).

[60] R. J. T. Nicholl, H. J. Conley, N. V. Lavrik, I. Vlassiouk, Y. S. Puzyrev, V. P. Sreenivas, S. T. Pantelides, and K. I. Bolotin, Nat. Commun. 6, 8789 (2015).

[61] R. J. T. Nicholl, N. V. Lavrik, I. Vlassiouk, B. R. Srijanto, and K. I. Bolotin, Phys. Rev. Lett. 118, 266101 (2017).

[62] H. B. Callen, Thermodynamics (Wiley, New York, 1960).

[63] W. Helfrich and R. M. Servuss, Nuovo Cimento D 3, 137 (1984).

[64] J.-B. Fournier and C. Barbetta, Phys. Rev. Lett. 100, 078103 (2008).

[65] J.-W. Jiang, J.-S. Wang, and B. Li, Phys. Rev. B 80, 205429 (2009).

[66] A. L. C. da Silva, L. Candido, J. N. Teixeira Rabelo, G. Q. Hai, and F. M. Peeters, Europhys. Lett. 107, 56004 (2014).

[67] D. Yoon, Y.-W. Son, and H. Cheong, Nano Lett. 11, 3227 (2011).

[68] W. Bao, F. Miao, Z. Chen, H. Zhang, W. Jang, C. Dames, and C. N. Lau, Nat. Nanotech. 4, 562 (2009).

[69] E. A. Kellett and B. P. Richards, J. Nucl. Mater. 12, 184 (1964).

[70] W. C. Morgan, Carbon 10, 73 (1972).

[71] B. Marsden, A. Mummery, and P. Mummery, Proc. Royal Soc. A 474, 20180075 (2018).

[72] N. W. Ashcroft and N. D. Mermin, Solid State Physics (Saunders College, Philadelphia, 1976).

[73] A. C. Bailey and B. Yates, J. Appl. Phys. 41, 5088 (1970).

[74] C. Kittel, Introduction to Solid State Physics (Wiley, New York, 1966).

[75] F. Behroozi, Langmuir 12, 2289 (1996).

[76] L. D. Landau and E. M. Lifshitz, Statistical Physics, 3rd ed. (Pergamon, Oxford, 1980).

[77] C. P. Herrero and R. Ramírez, Phys. Rev. B 97, 195433 (2018).

[78] O. L. Blakslee, D. G. Proctor, E. J. Seldin, G. B. Spence, and T. Weng, J. Appl. Phys. 41, 3373 (1970). 
[79] Y. Baskin and L. Meyer, Phys. Rev. 100, 544 (1955).

[80] C. P. Herrero, J. Phys.: Condens. Matter 20, 295230 (2008).

[81] R. Nicklow, N. Wakabayashi, and H. G. Smith, Phys. Rev. B 5, 4951 (1972).

[82] K. Komatsu, J. Phys. Chem. Solids 25, 707 (1964).

[83] J. C. Noya, C. P. Herrero, and R. Ramírez, Phys. Rev. B 53, 9869 (1996).

[84] R. Ramírez, C. P. Herrero, and E. R. Hernández, Phys. Rev. B 73, 245202 (2006).

[85] L. X. Benedict, S. G. Louie, and M. L. Cohen, Solid State Commun. 100, 177 (1996).

[86] T. Nihira and T. Iwata, Phys. Rev. B 68, 134305 (2003).
[87] K. C. Fong, E. E. Wollman, H. Ravi, W. Chen, A. A. Clerk, M. D. Shaw, H. G. Leduc, and K. C. Schwab, Phys. Rev. X 3, 041008 (2013).

[88] W. Desorbo and W. W. Tyler, J. Chem. Phys. 21, 1660 (1953).

[89] K. Komatsu and T. Nagamiya, J. Phys. Soc. Jpn. 6, 438 (1951).

[90] J. Krumhansl and H. Brooks, J. Chem. Phys. 21, 1663 (1953).

[91] P. G. Klemens, Aust. J. Phys. 6, 405 (1953).

[92] A. Lajevardipour, M. Neek-Amal, and F. M. Peeters, J. Phys.: Condens. Matter 24, 175303 (2012).

[93] V. N. Popov, Phys. Rev. B 66, 153408 (2002).

[94] J. Zimmermann, P. Pavone, and G. Cuniberti, Phys. Rev. B 78, 045410 (2008). 